\title{
Simulation of Bridging at the Static Surface Filtration by CFD- DEM Coupling
}

\author{
David Hund ${ }^{1, *}$, Sergiy Antonyuk ${ }^{1}$ and Siegfried Ripperger ${ }^{1,2}$ \\ ${ }^{1}$ Institute of Particle Process Engineering, University of Kaiserslautern, 67663 Kaiserslautern, Germany \\ ${ }^{2}$ IT for Engineering (it4e) GmbH, 67663 Kaiserslautern, Germany
}

\begin{abstract}
To achieve a better understanding of the bridging mechanism at the static surface filtration, simulations of particle deposition at a three-dimensional pore model of a filter were performed. The simulations are used to investigate the total filter resistance, which results from interferences between filter medium, particles and fluid. Thereby a CFD (Computational Fluid Dynamics) method for flow simulation was coupled with a Discrete Element Method (DEM) which calculates the particle interactions. The results show that a built particle bridge influences the flow through an unclosed pore. It was concluded that bridging of open pores is hindered at high velocities of approach caused by increasing percentage of closed pores. Moreover, it could be shown, that the total filter resistance depends on the approaching velocity and shape of particles.
\end{abstract}

\section{Introduction}

Solid-liquid filtration is the separation of dispersed particles from a fluid by means of filter media. At the static surface filtration the solid particles deposit on the surface of the filter medium. Further accumulation of particles lead to the filter cake build up. For the filtration processes, which are operating with short cycle times to increase the throughput, the filter resistance and the structure of the first particle layer are important characteristics. By the use of open-pore filter medium, the through-flow resistance can be reduced and the filtrate throughput can be increased. In such filtration processes the so called bridging can take place. Bridging means the pores are blocked by accumulated particles even though their diameters are smaller than the diameters of the pores of the filter medium (Fig. 1) [1, 2, 3].

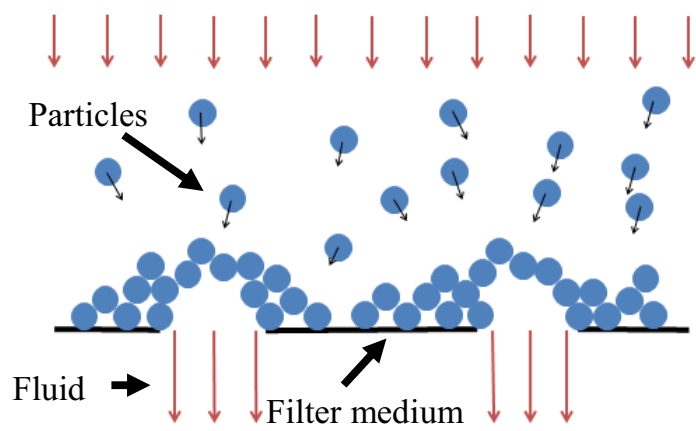

Fig. 1. Schematic illustration of the bridging mechanism

At the beginning of cake filtration processes, bridging is of particular importance, because it enables the cake building. Previous experimental studies have shown that the total filter medium resistance increases greatly as a result of interferences between the filter medium and the first particle layer [3]. In industrial filtration, this interference resistance can be 10 to 40 times the value of the unladen filter medium resistance. It contributes significantly to the total filter resistance [4].

In order to investigate the interference resistance and to understand the process of bridging, simulations for particle deposition were carried out. For this a simplified pore model was used considering particle-particle and particle-filter medium interactions. For this purpose, a CFD method to simulate the flow and a Discrete Element Method (DEM) to simulate the particle interactions were coupled.

\section{Simulation Methods}

\subsection{Discrete Element Method (DEM)}

The DEM, developed by Cundall and Strack in 1978 can be used to simulate the dynamics of particulate systems [5]. The method describes each particle in the system and its interactions with other particles or rigid walls (Fig. 2). Based on the force balance for particle $i$ the equation of translational motion (1) is solved.

$$
m_{i} \frac{\mathrm{d} \boldsymbol{v}_{i}}{\mathrm{~d} t}=\sum_{j=1}^{k_{i}} \boldsymbol{F}_{\mathrm{c}, i j}+\boldsymbol{F}_{\mathrm{g}, i}+\boldsymbol{F}_{\mathrm{d}, i}
$$

In the equations $m_{\mathrm{i}}$ and $\boldsymbol{v}_{\mathrm{i}}$ are the mass and the velocity of particles $i . \boldsymbol{F}_{\mathrm{c}, i j}$ are the contact forces between particles $i$ and $j$ and they are summed over the $k_{i}$ particles in contact with particle $i[5] . \boldsymbol{F}_{\mathrm{g}, i}$ is the gravitational force

\footnotetext{
David Hund: david.hund@mv.uni-kl.de
} 
and $\boldsymbol{F}_{\mathrm{d}, i}$ is the drag force acting on particle $i . \boldsymbol{F}_{\mathrm{d}, i}$ results from the interaction with the fluid.

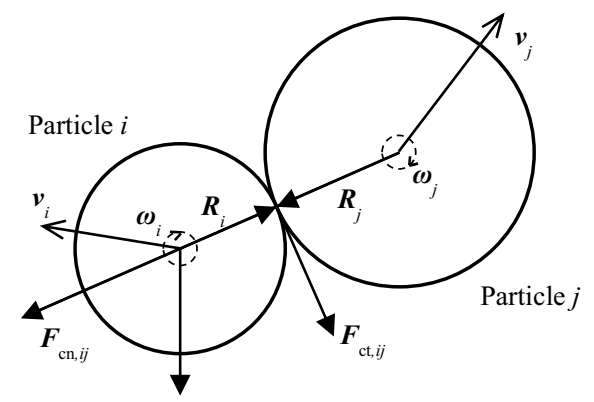

Fig. 2. Illustration of particle $i$ in contact with particle $j$

The contact force $\boldsymbol{F}_{\mathrm{c}, i j}$ between two particles can be decomposed into a normal $\boldsymbol{F}_{\mathrm{cn}, i j}$ and a tangential force $\boldsymbol{F}_{c t, i j \text {. }}$

$$
\boldsymbol{F}_{\mathrm{c}, i j}=\boldsymbol{F}_{\mathrm{cn}, i j}+\boldsymbol{F}_{\mathrm{ct}, i j}
$$

For the calculation of $\boldsymbol{F}_{\mathrm{cn}, i j}$ a non-linear model, based on Hertz [6] combined with a viscous damping term according to Tsuji [7] and adhesion according to the Johnson-Kendall-Roberts (JKR) theory [8] was used. The damping parameter $\alpha$ (Table.1) of the damping term [7] was determined based on the coefficient of restitution (COR) $e=0.5$. But the effective COR of the whole model depends on the impact velocity. $\boldsymbol{F}_{\mathrm{ct}, i j}$ is modelled based on the non-slip solution of the theory provided by Mindlin and Deresiewicz [9].

The contact forces $\boldsymbol{F}_{\mathrm{c}, i j}$ act at the contact point between particles $i$ and $j$. The lines of action of the contact forces which do not pass the centre of the particle will generate a torque $\boldsymbol{M}_{i j}$. It is given by:

$$
\boldsymbol{M}_{i j}=\boldsymbol{R}_{i} \times \boldsymbol{F}_{\mathrm{ct}, i j}
$$

where $\boldsymbol{R}_{i}$ is a vector from the mass centre of the particle to the contact point [4]. The governing equation for the rotational motion of particle $i$ with the moment of inertia $J_{i}$ is written as:

$$
J_{i} \frac{\mathrm{d} \omega_{i}}{\mathrm{~d} t}=\sum_{j=1}^{k_{i}} \boldsymbol{M}_{i j}
$$

where $\omega_{i}$ is the angular velocity of particle $i$.

\subsection{Governing equations in the CFD approach}

To consider that particles influence the fluid, the description of the flow in this work is made via modified Navier-Stokes equations. The averaged mass (1) and momentum (2) conservation equations are written as:

$$
\frac{\partial\left(\varepsilon \rho_{\mathrm{F}}\right)}{\partial t}+\nabla \cdot\left(\varepsilon \rho_{\mathrm{F}} \boldsymbol{u}\right)=0
$$

$\frac{\partial}{\partial t}\left(\rho_{\mathrm{F}} \varepsilon \boldsymbol{u}\right)+\nabla \cdot\left(\rho_{\mathrm{F}} \varepsilon \boldsymbol{u} \boldsymbol{u}\right)=-\nabla p+\nabla \cdot(\varepsilon \vec{\tau})+\varepsilon \rho_{\mathrm{F}} \mathbf{g}-\boldsymbol{S}$

with the fluid volume fraction $\varepsilon$, the averaged velocity $\boldsymbol{u}$, the density $\rho_{F}$ of the fluid, the gravity $\mathbf{g}$ and the viscous stress tensor $\bar{\tau}$. $S$ is an external volumetric force which considers the drag force of the particles on the fluid.

\subsection{CFD-DEM coupling}

In this work, a four way coupling was used which means that particle-fluid, fluid-particle, particle-particle and particle-wall interactions were considered. In Fig. 2 is shown schematically the coupling of CFD and DEM. In the first step the conservation equations are solved by the CFD. Afterwards the flow parameters are transferred to DEM to calculate the hydrodynamic forces acting on the particles. Then the balances of forces for each particle are solved and the new velocity and the new position of the particles are calculated. Finally the fluid flow is computed again by CFD depending on the new fluid volume fraction of the mesh cell and the source term $S$. In general the source term $S$ considers all fluid forces acting on the particle. In the present work only the drag force of all particles $\boldsymbol{F}_{\mathrm{d}}$ is considered.

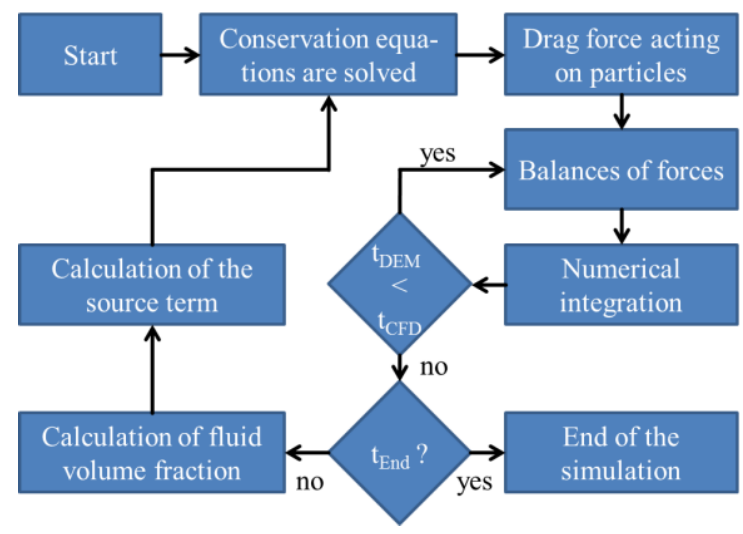

Fig. 3. Simulation flowchart of CFD-DEM coupling

The drag force on an isolated particle is calculated by the following equation [10]:

$$
\boldsymbol{F}_{\mathrm{d}}=\frac{\rho_{\mathrm{F}}}{2} c_{\mathrm{d}}\left(\operatorname{Re}_{\mathrm{P}}\right) A_{\mathrm{P}}(\boldsymbol{u}-\boldsymbol{v})|\boldsymbol{u}-\boldsymbol{v}|
$$

where $c_{\mathrm{D}}$ is the drag coefficient and $A_{\mathrm{P}}$ the cross section of the particle. Depending on the particle Reynolds number $\operatorname{Re}_{\mathrm{P}} c_{\mathrm{D}}$ is defined according to Stokes, Schiller and Naumann [11] and Newton [12] respectively.

$$
\operatorname{Re}_{\mathrm{P}}=\frac{\rho_{F} \varepsilon|\boldsymbol{u}-\boldsymbol{v}| d_{\mathrm{P}}}{\eta}
$$

$$
\begin{array}{lrr}
\text { if } & \operatorname{Re}_{\mathrm{P}} \leq 0.5 \quad c_{\mathrm{d}}=\frac{24}{\mathrm{Re}_{\mathrm{P}}} \\
\text { if } & 0.5<\mathrm{Re}_{\mathrm{P}} \leq 1000 \quad c_{\mathrm{d}}=\frac{24\left(1+0.15 \mathrm{Re}_{\mathrm{P}}^{0,687}\right)}{\operatorname{Re}_{\mathrm{P}}} \\
\text { if } & 10^{5}>\mathrm{Re}_{\mathrm{P}}>1000 \quad c_{\mathrm{d}}=0.44
\end{array}
$$

\section{Results}

\subsection{Pore model and simulation set up}

The filter medium, as shown in Fig. 3, is represented by a simplified pore model with cylindrical pores and a pore diameter of $d_{\text {Pore }}=20 \mu \mathrm{m}$. The suspension flows through the filter medium perpendicular. Besides the bridging at 
a single pore, the influence of a blocked pore on the bridging at surrounding pores is considered. The pore model includes four pores. To exclude boundary effects periodic boundary conditions were used.

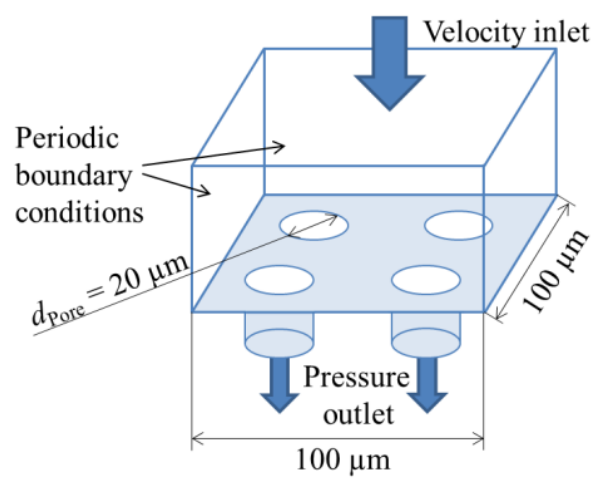

Fig. 4. Simulation model with fur cylindrical pores

In this work, the bridging formation on both spherical and cylindrical polyamide particles is investigated (Fig. 4). The two particle systems are identical in volume.
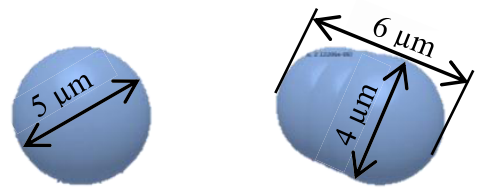

Fig. 5. Spherical (left) and non-spherical (right) particles

In DEM the non-spherical particles were modelled with the multi-sphere approach [12]. In this approach three smaller spherical particles were connected in order to form a cylindrical particle. In Table 1 all necessary simulation parameters are shown. Further information are given in the corresponding literature.

Table 1. Simulation setup

\begin{tabular}{ll}
\hline Pore Model & \\
\hline Side length & $L_{\mathrm{S}}=100 \mu \mathrm{m}$ \\
Pore Diameter & $D_{\text {Pore }}=20 \mu \mathrm{m}$ \\
\hline & \\
Particle phase & \\
\hline Particle size & $d_{\mathrm{P}}=5 \mu \mathrm{m}$ \\
$\quad$ Spherical & $L_{\mathrm{P}}=6 \mu \mathrm{m}, H_{\mathrm{P}}=4 \mu \mathrm{m}$ \\
$\quad$ Non-spherical & $\rho_{\mathrm{P}}=1180 \mathrm{~kg} / \mathrm{m}^{3}$ \\
Particle density & $G=0.89 \mathrm{GPa}$ \\
Shear modulus [6, 7] & $E=2.5 \mathrm{GPa}$ \\
Young's modulus [6, 7] & $v=0.4$ \\
Poisson ratio [6, 7] & $\mu=0.5$ \\
Friction coefficient [9] & $\alpha=1.728$ \\
Damping parameter [7] & $\gamma=0.41 \mathrm{~J} / \mathrm{m}^{2}$ \\
Surface energy [8] & \\
\hline & \\
Fluid phase & $\rho_{\mathrm{F}}=998.2 \mathrm{~kg} / \mathrm{m}^{3}$ \\
\hline Density & $\eta_{\mathrm{F}}=0.001 \mathrm{~Pa} \cdot \mathrm{s}$ \\
Viscosity &
\end{tabular}

\subsection{Bridging mechanism}

The bridging simulations were performed in dead-end mode. Either a constant pressure difference or a constant specific filtrate volume flow was prescribed. The simulations were terminated if the pores were blogged by the particles. The bridge formation is initially discussed at a constant flow velocity of $u=0.5 \mathrm{~m} / \mathrm{s}$ and at a volume concentration of the particles in the fluid of $c_{\mathrm{v}}=0.1 \%$. In Fig. 5 both pictures (left and right) show a section through two of four pores. On the left side the volume fraction and on the right the associated particle velocity is illustrated. It is clearly indicated that the particles first deposited around the pores and that the process of bridging is still not completed.
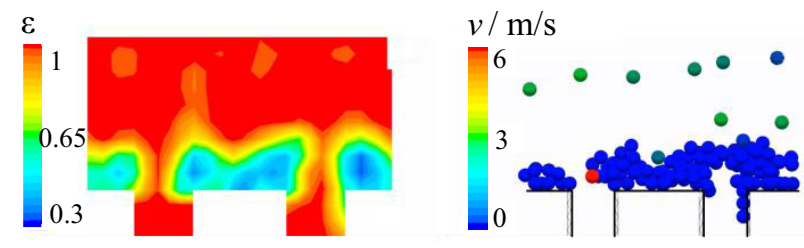

Fig. 6. Volume fraction of fluid $\varepsilon$ while bridging (left) and the associated particle velocity $v$ (right)

To figure out the influence of a clogged pore on the flow through the other pores the specific flow rate of each pore is presented in Fig. 6. First, the flow rate decreases in pore 3 because of deposited particles. As a consequence the averaged flow rates in the other pores increase due to the constant flow rate at the inlet. After $t=3 \mathrm{~ms}$ pores 1,3 and 4 are blogged and only pore 2 is unclosed. After $t=4.3 \mathrm{~ms}$ even pore 2 is clogged and the specific flow rate in each pore is constant. Because a nearly built bridge over pore 2 collapses at $t=2.3 \mathrm{~ms}$ the flow rate in pore 2 increases abruptly.

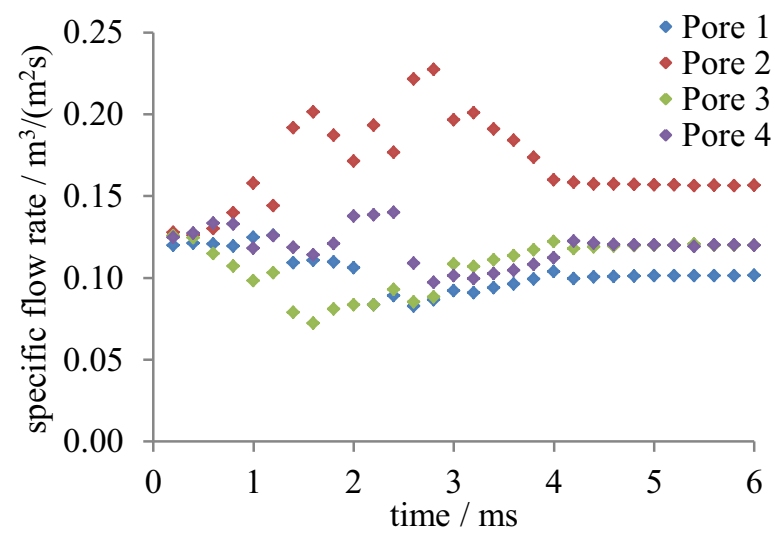

Fig. 7. Specific flow rate in each pore at a constant flow velocity at the inlet $u=0.5 \mathrm{~m} / \mathrm{s}, c_{\mathrm{v}}=0.1 \%, d_{\mathrm{P}}=5 \mu \mathrm{m}$

Fig. 7 shows the corresponding pressure drop across the filter medium over the time. At $t=0.1 \mathrm{~ms}$ the pressure drop results from the flow resistance of the unladen filter medium at a constant flow velocity $u=0.5 \mathrm{~m} / \mathrm{s}$. Afterwards the pressure drop increases because of deposited particles around the pores and clogging of pores. 


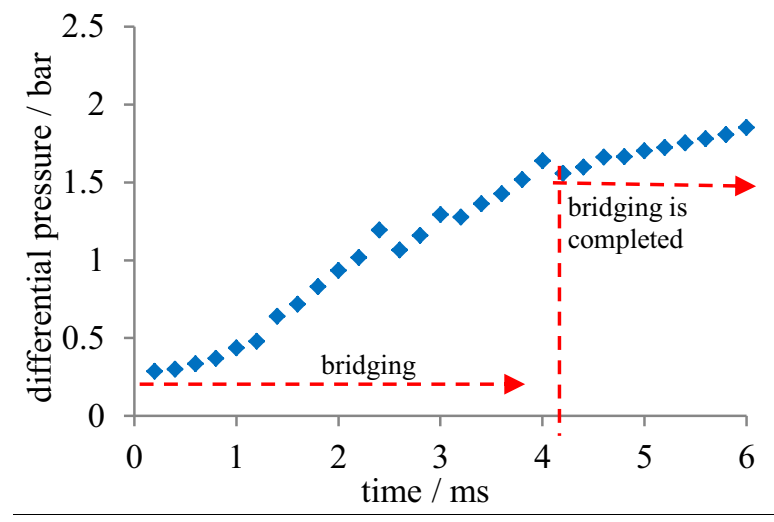

Fig. 8. Pressure drop across the filter medium at a constant flow velocity at the inlet $u=0.5 \mathrm{~m} / \mathrm{s}, c_{\mathrm{v}}=0.1 \%, d_{\mathrm{P}}=5 \mu \mathrm{m}$

As well as presented in Fig. 6 at $t=2.3 \mathrm{~ms}$ the pressure drop decreases because of a collapsed bridge. If all pores are clogged the filter cake build up begins and the pressure drop increases linearly.

Fig. 8 illustrates the filter cake height over time for different specific flow rates. If the filter cake height increases linearly with time bridging is finished. It can be seen that the higher the flow velocity the later bridging is completed. Because of high drag forces acting on particles, at a flow velocity of $u=2 \mathrm{~m} / \mathrm{s}$ no bridging takes place.

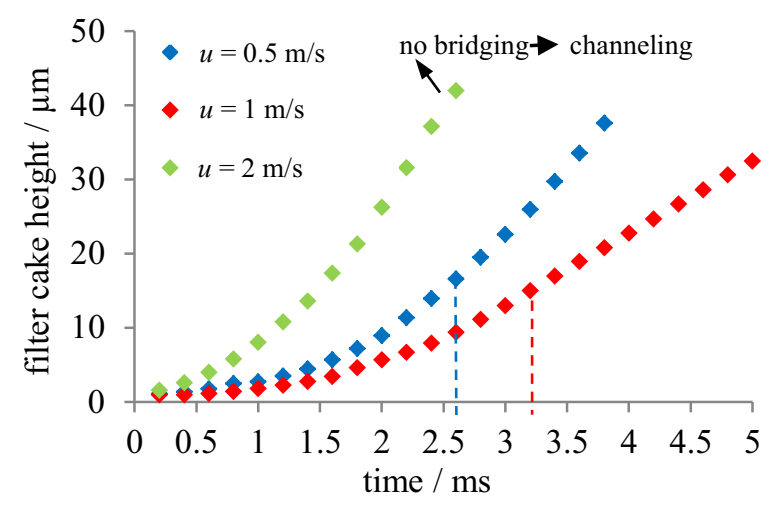

Fig. 9. Filter cake height over time for different specific flow rates $u=0.5 \mathrm{~m} / \mathrm{s}, u=1 \mathrm{~m} / \mathrm{s}, u=2 \mathrm{~m} / \mathrm{s}, c_{\mathrm{v}}=0.1 \%, d_{\mathrm{P}}=5 \mu \mathrm{m}$

In Fig. 9 the corresponded volume fractions of fluid above the pores are shown. As can be seen the higher the flow velocity the higher the built bridge and at a flow velocity of $u=2 \mathrm{~m} / \mathrm{s}$ continuous channels are formed.

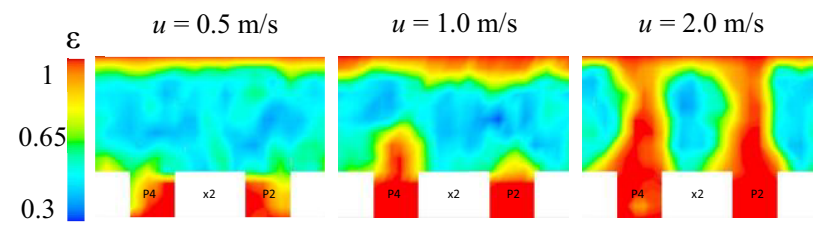

Fig. 10. Volume fraction $\varepsilon$ of fluid at different flow velocities $u=0.5 \mathrm{~m} / \mathrm{s}, u=1 \mathrm{~m} / \mathrm{s}, u=2 \mathrm{~m} / \mathrm{s}, c_{\mathrm{v}}=0.1 \%, d_{\mathrm{P}}=5 \mu \mathrm{m}$

Finally the bridging mechanism with different shapes of particles (Fig. 4) at a constant pressure drop will be discussed. The filter cake height over time is presented in Fig. 10. The results show that bridging at nonspherical particles is completed earlier. The cylindrical particles are better placed together. Due to the higher porosity in the filter cake of the non-spherical particles, the slope in the linear region is higher. Thus the filter cake build up at non-spherical particles is faster than with spherical particles.

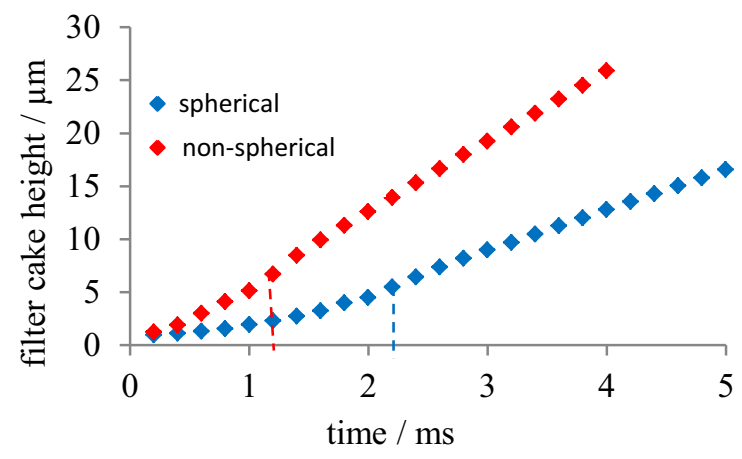

Fig. 11. Filter cake height over time for different particle shapes, $\Delta p=0.5$ bar, $c_{\mathrm{v}}=0.1 \%$

\section{Conclusions}

In this manuscript a CFD-DEM coupled simulation method was introduced. The method appears to describe and investigate the influence of built bridges on the flow through of unclosed pores. It was found out that bridging in open pores is hindered at high velocities of approach caused by increasing percentage of closed pores. Further could be shown that the shape of particles influences the time until the pores are clogged. Compared to the spherical particles the cylindrical particles which were modelled using the multi-sphere approach clog the pore first.

\section{References}

1. G. Agbangla, E. Climent, P. Baccin, Sep Purif Technol, 101, 42-48 (2012)

2. C. Schnitzer, S. Ripperger, Filtr. und Sep. 2, 58-60 (2009)

3. A. Rushton, The Chem. Eng., (1970)

4. J.W. Tichy, VCH-Verlag, (2007)

5. P.A. Cundall, O.D. Strack, Geotechnique 29, 47-65 (1979)

6. H. Hertz, J reine angew Math, 92, 156-171 (1882)

7. Y. Tsuji, T. Tanaka, T. Ishida, Powder Technol. 71, 239-250 (1992)

8. K.L. Johnson, K. Kendall, D. Roberts, Proc R. Soc. Lond. 324, 301-313 (1971)

9. R. D. Mindlin, H. Deresiewicz, Adv energy syst div, 327-344 (1953)

10. Lord Rayleigh, C R, 159, 109-110 (1913)

11. L. Schiller, A. Naumann, Z Ver Deutsch Ing 77, (318-320) 1935

12. R. Johnson, Handbook of Fluid Dynamics, 2016

13. J. Favier et al., Eng. Comput. 16, 467-480 (1999) 